\title{
Do quantitative cultures of protected brush specimens modify the initial empirical therapy in ventilated patients with suspected pneumonia?
}

\author{
F. Rodríguez de Castro*, J. Solé-Violán**, A. Aranda León**, \\ J. Blanco López**, G. Julià-Serdà*, P. Cabrera Navarro*, J. Bolaños Guerra**
}

Do quantitative cultures of protected brush specimens modify the initial empirical therapy in ventilated patients with suspected pneumonia? F. Rodríguez de Castro, J. SoléViolán, A. Aranda León, J. Blanco López, G. Julià-Serdà, P. Cabrera Navarro, J. Bolaños Guerra. CERS Journals Ltd 1996.

ABSTRACT: The real contribution of the protected specimen brush (PSB) to guide the choice of antimicrobial therapy in ventilated patients with severe pneumonia is not well established. This study aimed to assess how data obtained by PSB changed the initial empirical therapy.

One hundred and ten ventilated patients with suspected pneumonia were entered into a prospective study. All patients underwent fibreoptic bronchoscopy within the first $24 \mathrm{~h}$ after clinical suspicion of pneumonia. Specimens were obtained by PSB and were processed for quantitative cultures using standard methods. When patients were started on empirical antibiotics, a standard therapeutic regimen was followed. The decision to introduce, withdraw, maintain, or modify antibiotic therapy, according to the PSB culture results, was left to the attending physician.

Pneumonia was the final diagnosis in $45(41 \%)$ of the 110 patients. Pneumonia was excluded in $47(43 \%)$ patients and in the remaining $18(16 \%)$ the diagnosis was uncertain. The initial therapeutic plans were considered to be adequate in $\mathbf{4 0}$ patients $(36 \%)$ and inadequate in 37 cases (34\%). In 17 patients (15\%), therapeutic changes were made based on the results of PSB cultures. In one case appropriate antibiotics were introduced; in nine cases the therapeutic regimen was reduced to select a narrower and rational therapy; and in seven patients therapy was changed because the prescribed antimicrobial agents were ineffective against the organisms recovered.

This study shows that therapeutic changes were made based on the results of PSB cultures in $38 \%$ of the patients with pneumonia. In contrast, the PSB technique has no impact on antimicrobial therapy of patients without pneumonia.

Eur Respir J., 1996, 9, 37-41.

\begin{abstract}
* Section of Pneumology and **Intensive Care Unit, Hospital Universitario "Ntra. Sra. del Pino", Facultad de Medicina, Universidad de Las Palmas de Gran Canaria (ULPGC), Las Palmas de Gran Canaria, Spain.
\end{abstract}

Correspondence: F. Rodríguez de Castro Sección de Neumología

Hospital Universitario "Ntra. Sra. del Pino" Angel Guimerá 93

35005 Las Palmas de Gran Canaria Spain

Keywords: Antibiotics protected specimen brush severe pneumonia

Received: December 191994 Accepted after revision October 171995
Bacterial pneumonia is a frequent complication in mechanically-ventilated patients and a major contributor to overall morbidity and mortality in the Intensive Care Unit (ICU) [1, 2]. Several reports have indicated that information on the infectious status of patients on ventilators with pulmonary infiltrates can be obtained by using quantitative cultures of samples recovered by protected specimen brush (PSB) and bronchoalveolar lavage (BAL) [3-11]. What is missing is a clear knowledge of how the data can be employed to aid in patient management $[12,13]$. Are the data useful in medical decision making with respect to maintaining or changing antibiotics? Therefore, we have performed a prospective study to evaluate the accuracy of the therapeutic plans for severe pneumonia usually employed in our unit and the contribution of quantitative cultures of PSB specimens to guide the choice of antimicrobial treatment.

\section{Methods}

Over a 25 month period, from June 1992 to July 1994, 110 immunocompetent, intubated and mechanicallyventilated patients entered a prospective study. Patients were eligible for the study if they fulfilled the following criteria: fever $>38.5^{\circ} \mathrm{C}$; presence of purulent tracheobronchial secretions; leucocytosis $\left(>12,000\right.$ cells $\cdot \mathrm{mm}^{-3}$, $\left.>12 \times 10^{9} \cdot \mathrm{L}^{-1}\right)$ or leucopenia $\left(<4,000\right.$ cells $\left.\cdot \mathrm{mm}^{-3},<4 \times 10^{9} \cdot \mathrm{L}^{-1}\right)$ and new infiltrates on chest roentgenogram. When patients showed at least three of these criteria, radiographic infiltrates being always present, a fibreoptic bronchoscopy was performed within the first $24 \mathrm{~h}$ after the clinical diagnosis of pneumonia was suspected.

The procedure was performed whilst patients were ventilated with $100 \%$ oxygen, without positive end-expiratory pressure and having continuous finger pulse oximetry 
(Ohmeda Biox, 3740 Louisville, CO, USA) and electrocardiographic monitoring. The fibreoptic bronchoscope (Olympus BF-P20D; Olympus Optical Corp. of America, New Hyde Park, NY, USA) was introduced into the trachea through an endotracheal tube of $\geq 8 \mathrm{~mm}$ diameter via a sterile connector (Bodai Suction Safe. Y: Sontek Medical, Lexington, MA, USA) and advanced next to the orifice of the sampling area to visualize the entrance to the radiographically abnormal bronchial subsegment. The telescoping plugged catheter (MBB 120388-5504; Mill-Rose Laboratories, Mentor, OH, USA) was inserted through the inner suction channel and advanced, under direct vision, to a wedge peripheral position to obtain lower airway secretions, according to the technique described by Wimberley et al. [14]. No suction was applied before taking the specimen and no local anaesthetic agents were used.

The brush was aseptically cut into a vial containing 1 $\mathrm{mL}$ of sterile $0.9 \%$ saline. The vial was then vigorously mixed using a vortex mixer for at least $60 \mathrm{~s}$ to thoroughly suspend all material from the brush. Specimens were immediately delivered to the laboratory and processed for quantitative bacterial and fungal culture using standard methods, as has been described in previous studies [9-11, 15]. Bacterial counts of $\geq 10^{3}$ colony forming units $(\mathrm{cfu}) \cdot \mathrm{mL}^{-1}$ were used as the cut-off point to establish a positive result.

The time without antibiotics and the type, reason and duration of treatment received before the procedure were recorded. When patients were started on empirical antibiotics for a presumptive diagnosis of pneumonia (i.e. a few hours before or immediately after the bronchoscopic procedure), the following therapeutic plans were used: 1) severe community-acquired pneumonia: cefotaxime plus erythromycin; 2) early-onset pneumonia (present at, or before, the seventh day of intubation): cefotaxime plus an aminoglycoside agent; 3 ) early-onset pneumonia in patients with impaired consciousness on admission: cefotaxime plus vancomycin or plus clindamycin; 4) late pneumonia (developed more than 7 days after intubation): an aminoglycoside plus ceftazidime or plus piperacillin.

In patients already receiving antimicrobials, the decision to withdraw, maintain, or modify antibiotic therapy, according to the PSB culture results, was left to the attending physician. The initial therapeutic strategy was considered as inadequate if: 1) antibiotics were inactive on micro-organisms recovered by PSB in significant counts; 2) only a single agent was active against multiresistant isolated bacteria; 3) antimicrobial drugs were unnecessary because the patient did not have pneumonia; or 4) no antimicrobial treatment was administered to patients with pneumonia.

Definite diagnosis of pneumonia $(n=45)$ was based on: 1) identification of consolidated foci and polymorphonuclear leucocyte accumulation in bronchioles and adjacent alveoli at autopsy performed within 5 days after the bronchoscopic procedure $(n=3) ; 2)$ a positive result of blood and/or pleural fluid culture without an extrapulmonary focus of infection $(n=16)$; 3) a rapid cavitation of the lung infiltrate $(\mathrm{n}=6)$; and 4$)$ a clinical outcome consistent with bacterial pneumonia whilst receiving appropriate antibiotic therapy for the organisms cultured in PSB in significant growth $(n=33)$.

Pneumonia was considered definitely excluded $(n=47)$ if at least one of the following criteria was fulfilled: 1) full recovery without appropriate antimicrobial therapy $(n=29)$, or without changes in the antibiotic therapy initiated at least $72 \mathrm{~h}$ before the appearance of infiltrates $(n=12)$ with alternate explanations for the findings, such as atelectasis or pulmonary oedema; and 2) no signs of bacterial pneumonia at autopsy performed within 5 days after the bronchoscopic procedure $(n=6)$.

Moreover, subsequent changes in clinical course, radiographic findings and modifications in antibiotic therapy were recorded. Atelectasis was diagnosed when complete resolution of the infiltrates occurred during the first $48 \mathrm{~h}$ after its appearance. Cardiogenic and noncardiogenic pulmonary oedema was diagnosed by pulmonary arterial catheterization and response to appropriate therapy.

The number of organisms recovered from PSB is expressed as $\mathrm{cfu} \cdot \mathrm{mL}^{-1}$. Other results are expressed as mean \pm standard deviation or as a percent of total patients. Determinations of sensitivity and specificity were performed using standard methods [16]. The Mann-Whitney U test was used for intergroup comparisons. A p-value of less than 0.05 was considered significant.

\section{Results}

The study population consisted of 110 mechanicallyventilated patients ( 74 males and 36 females) with a mean age of $49 \pm 19$ yrs (range 14-83 yrs). Patients had been admitted to the ICU and required ventilatory assistance because of respiratory failure, impaired consciousness or loss of muscular strength as a result of neurological disorders $(n=29)$ (head injury 14, cerebrovascular disease 7 , drug overdose 5, status epilepticus 2, meningitis 1); postoperative respiratory insufficiency $(n=32)$ (heart surgery 11 , thoracic surgery 8 , abdominal surgery 7 , neurosurgery 6); acute exacerbation of $\operatorname{COPD}(n=6)$; severe community-acquired pneumonia $(n=1)$; multiple trauma $(n=14)$; heart failure $(n=14)$; and miscellaneous conditions $(n=14)$. The mean length of time during which patients were mechanically-ventilated was $8.5 \pm 11.7$ days (range 1-71 days). Forty seven patients (45\%) had been receiving antibiotics for an average of $6.7 \pm 6.8$ days (range 1-28 days), but in all except 12 cases, antibiotic therapy was stopped at least $48 \mathrm{~h}$ before fibreoptic bronchoscopy.

Pneumonia was the final diagnosis in $45(41 \%)$ of the 110 patients. In 47 (43\%) patients pneumonia was excluded and in the remaining $18(16 \%)$ the diagnosis could not be accurately defined. PSB yielded significant bacterial growth $\left(\geq 10^{3} \mathrm{cfu} \cdot \mathrm{mL}^{-1}\right)$ in 33 out of 45 patients with pneumonia (73\%), nonsignificant growth in three, and was sterile in nine. In the 47 patients without pneumonia, PSB cultures were sterile in 40 and exhibited growth of micro-organisms in concentrations lower than $10^{3}$ $\mathrm{cfu} \cdot \mathrm{mL}^{-1}$ in seven.

All but 12 patients were off long-term antibiotic therapy when bronchoscopy was carried out. The clinical condition of 26 patients made it possible to wait for the 
microbiological results of PSB before deciding whether or not to introduce antibiotics, although the ultimate decision was always left to the best opinion of the attending physician. In fact, despite obtaining only one positive microbiological result, 19 out of these 26 cases were finally treated and only seven remained off antibiotics. Seventy two patients (44 with pneumonia and 28 without pneumonia) were started on empirical antibiotics according to the standard regimen in the peribronchoscopic period ( 2 or $3 \mathrm{~h}$ before or immediately after the bronchoscopy). In 22 patients who did not have pneumonia, this empirical antibiotic treatment was promptly discontinued based on clinical and radiographic findings in the subsequent follow-up, rather than on the results of PSB. Sixteen patients developed early pneumonia; 18 had late nosocomial pneumonia; 10 patients developed early-onset pneumonia whilst ventilated because of impaired consciousness; and one was admitted to the ICU because of severe community-acquired pneumonia.

The aetiological agents of pneumonia are shown in table 1. Haemophilus influenzae (13), Pseudomonas aeruginosa (7) and Acinetobacter calcoaceticus (4) were the Gram-negative organisms most often isolated, accounting for the 24, 13 and $7 \%$ of the total causative organisms (55), respectively. The most frequently isolated

Table 1 - Micro-organisms $(n=55)$ isolated in 45 patients with pneumonia

\begin{tabular}{lll}
\hline Pneumonia & Micro-organism & $\mathrm{n}$ \\
\hline Community-acquired pneumonia $(\mathrm{n}=1)$ & S. pneumoniae & 1 \\
Early nosocomial pneumoniae $(\mathrm{n}=16)$ & H. influenzae & 5 \\
& S. pneumoniae & 4 \\
& S. aureus & 2 \\
& E. coli & 2 \\
& P. aeruginosa & 1 \\
& M. morgagni & 1 \\
& P. mirabilis & 1 \\
Early nosocomial pneumonia and & H. influenzae & 6 \\
impaired consciousness (n=10) & S. aureus & 3 \\
& S. pneumoniae & 2 \\
& S. viridans & 2 \\
& E. cloacae & 1 \\
Late nosocomial pneumonia (n=15)* $*$. coli & 1 \\
& P. aeruginosa & 6 \\
& A. calcoaceticus & 4 \\
& S. aureus & 2 \\
& H. influenzae & 2 \\
& K. influenzae & 2 \\
& S. marcescens & 2 \\
& S. pneumoniae & 1 \\
& P. cepacea & 1 \\
& P. mirabilis & 1 \\
& E. cloacae & 1 \\
& E. coli & 1 \\
\hline & &
\end{tabular}

*: three pneumonias were not diagnosed on microbiological basis. H. influenzae: Haemophilus influenzae; $P$. aeruginosa: Pseudomonas aeruginosa; A. calcoaceticus: Acinetobacter calcoaceticus; S. pneumoniae: Streptococcus pneumoniae; S. aureus: Staphylococcus aureus; S. marcescens: Serratia marcescens; E. coli: Escherichia coli; S. viridans: Streptococcus viridans; K. pneumoniae: Klebsiella pneumoniae; E. cloacae: Enterobacter cloacae; M. morgagni: Morganella morgagni; P. mirabilis: Proteus mirabilis; P. cepacea: Pseudomonas cepacea.
Gram-positive organisms were Streptococcus pneumoniae (eight cases, 15\%) and Staphylococcus aureus (seven cases, 13\%). Pneumonia was polymicrobial in 11 cases $(24 \%)$ and there were three pneumonias that were not diagnosed on microbiological basis (necropsy findings in two, and rapid cavitation of pulmonary infiltrates in one patient).

In forty out of 110 patients (36\%), the initial therapeutic plans were considered adequate, either because antibiotics were not started in the group of patients without pneumonia $(n=7)$, or because the micro-organisms isolated by either PSB $(n=18)$, blood and/or pleural fluid $(n=8)$, or both $(n=7)$, in patients with pneumonia were adequately treated by the selected regimen. In contrast, initial therapeutic plans were considered inadequate in 37 cases (34\%) (table 2): in one infected patient because antibiotics were not started empirically; eight patients with pneumonia because the initial empirical therapy was not effective on the recovered organisms (seven from PSB and one from blood culture); and in 28 patients who did not have pneumonia and received empirical antimicrobial agents. In the remaining 33 patients, the adequacy or inadequacy of the initial therapeutic plan could not be defined: there were 12 cases (all belonging to the nonpneumonia group) in which no new therapeutic plan was formulated because antibiotics, initiated at least $72 \mathrm{~h}$ before the bronchoscopic procedure, were not ended; in the patients with pneumonia of unknown aetiology $(n=3)$, no modification of the initial therapeutic plan was carried out; and, finally, there were 18 patients in whom antibiotics were started despite a negative microbiological result of PSB. In these patients a definite diagnosis could not be accurately established because they did not meet any of the above-mentioned criteria of pneumonia and, in those who died, an autopsy was performed more than 5 days after the bronchoscopic procedure or it was denied.

In 17 patients $(15 \%)$, therapeutic changes were made based on the results of PSB cultures. In one case, appropriate antibiotics were introduced; in nine cases the therapeutic regimen was reduced to select a narrower and more rational therapy; and in seven patients therapy was changed because the prescribed antimicrobial agents were ineffective against the organisms recovered. In this latter group the microorganisms involved were Acinetobacter calcoaceticus (4), Staphylococcus aureus (2), Pseudomonas aeruginosa (1) and Serratia marcescens (1). With respect

Table 2. - Causes of inadequate initial empirical strategy

\begin{tabular}{|c|c|c|c|}
\hline \multicolumn{4}{|c|}{$\begin{array}{l}\text { Therapeutic plan inadequate } \\
\qquad \mathrm{n}=37 \quad(34 \%)\end{array}$} \\
\hline No pneumonia & \multicolumn{3}{|c|}{ Pneumonia } \\
\hline \multirow[t]{2}{*}{$\begin{array}{c}\text { Treated with ATB } \\
n=28\end{array}$} & & & $\begin{array}{c}\text { ATB not } \\
\text { started } \\
\text { empirically }\end{array}$ \\
\hline & $\begin{array}{c}\mathrm{PSB}^{\dagger} \\
\mathrm{n}=7\end{array}$ & $\begin{array}{l}\mathrm{BC}^{\dagger} \\
\mathrm{n}=1\end{array}$ & \\
\hline
\end{tabular}

ATB: antibiotics; PSB protected specimen brush; BC: blood cultures. + specimen on which change was based. 
to changing therapeutic plans, we have not found any significant association when age or days of mechanical ventilation among patients with pneumonia were considered.

No complications related to PSB use were encountered during this study.

\section{Discussion}

The aim of this study was to evaluate the usefulness of PSB, in terms of modifying a standard antibiotic regimen. The study showed that in 17 out of the 110 patients (25\%), therapeutic changes were made based on the results of PSB cultures. These comprised $38 \%$ of the 45 patients with a final diagnosis of pneumonia. On the other hand, according to our results, the PSB technique had no impact on the antimicrobial therapy of patients without pneumonia.

There have been few studies in which the clinical usefulness of quantitative cultures of PSB specimens has been prospectively assessed. In a study by FAGON and co-workers [17], empirical therapeutic plans were found to be inappropriate in $58 \%$ of patients, either because antibiotics were not used or because organisms responsible for pneumonia were not sensitive to the selected regimen. More recently, the same group [18] found that only $33 \%$ of therapeutic plans proposed for patients with suspected nosocomial pneumonia represented effective therapy.

We have conducted a prospective study in which patients with clinical pneumonia have been allocated to receive conventional empirical antibiotic regimens to determine the role of PSB data in managing these patients. We have observed that pneumonia was accurately diagnosed in only 45 of 110 cases, reflecting the difficulty in distinguishing pneumonia on clinical grounds alone. Since PSB culture results are not available for $24-48 \mathrm{~h}$, it was not helpful in making the initial decisions of whether therapy was required and which antibiotics were appropriate. Thus, 44 of 45 patients who had pneumonia, and 28 of 47 patients who did not have pneumonia were initially treated according to previously established and widely accepted therapeutic strategy [19-22]. These therapeutic regimens were also supported by our previous knowledge of the most frequent organisms isolated in our unit [10].

According to our empirical therapeutic plans and allowing for the microbiological results obtained, cefotaxime alone would have adequately covered most of the microorganisms causing early-onset pneumonias (all but one case of $P$. aeruginosa pneumonia). Moreover, two "unexpected" cases of $S$. aureus pneumonia in this group would also have been properly treated with cefotaxime. Our data show that the introduction of vancomycin in the aspiration induced early-onset pneumonia is not indispensable, because all the $S$. aureus pneumonias in this group $(30 \%)$ were methicillin-sensitive. However, the prevalence of methicillin-resistant $S$. aureus in our hospital has risen from 3\% in 1992 to $12 \%$ in 1994. Moreover, therapeutic changes based on an ineffective antibiotic regimen against the organisms recovered by PSB included two cases of methicillin-resistant $S$. aureus, belonging to the late-onset pneumonia group.

Some limitations of this study must be considered before the results can be generalized to clinical practice. Considering the PSB technique, patient therapy may be affected in two ways: 1) clinicians may prescribe more appropriate antibiotics for patients who need them (patients who have pneumonia); or, 2) clinicians may withhold antibiotics in patients who do not have pneumonia. Even if the outcome of an individual patient without pneumonia may not dramatically improve by withholding antibiotics in that patient, empirical broad-spectrum antibiotic therapy is not without risk. It induces drug toxicity and adverse drug reactions [23], the development of resistant flora and superinfection [24]. Furthermore the total cost involved in managing these patients is increased [5]. In addition, to falsely attribute fever, leucocytosis and pulmonary infiltrates to pneumonia may also lead to potentially fatal misdiagnosis in these critically ill patients. Therefore, to be able to demonstrate this potential advantage (i.e. to withhold antibiotic therapy in patients without pneumonia) of the PSB technique over a strategy based only on clinical evaluation would have been important. Obviously, this would have been possible only if the attending physicians had discontinued antimicrobial agents when the test results were negative. However, as we have found in other works [9-11], the use of the PSB gave a significant percentage of false-negative results (12 out of 45). Considering this diagnostic sensitivity, there would remain a risk that basing antibiotic therapy on nonsignificant PSB results might lead to a substantial proportion of patients with pneumonia not receiving appropriate therapy. Therefore, in our study, a negative PSB result usually led to maintaining empirical antibiotics and, therefore, unnecessary antibiotic therapy was not always avoided by PSB cultures. Moreover, purulent tracheobronchitis is one of the most frequent nosocomial infections in intubated patients and it would probably require antibiotics, even though PSB cultures were negative.

Another limitation of the study design is that of the 45 patients considered to have pneumonia, only 18 developed late nosocomial pneumonia. All other patients developed early-onset infection and one patient was included in the study because of community-acquired pneumonia. This fact could explain why empirical treatment was frequently effective in these patients. It is much harder to determine empirically an effective treatment in patients with late-onset pneumonia. However, we have not found any significant association between changing therapeutic plans and days of mechanical ventilation among patients with pneumonia. The PSB technique might be higher in a different study population.

Some issues still remain to be resolved. Whether the benefits of using PSB compensate for its potential negative consequences of either not treating some patients with pneumonia, or not avoiding unnecessary antibiotics in noninfected patients and its costs, is difficult to assess. Furthermore, a randomized study would be required to clarify whether specific antimicrobial treatment produces a better patient outcome than does empirical therapy. 


\section{References}

1. Penington JE. Nosocomial respiratory infections. In: Mandell GL, Douglas RG, Bennett JE, eds. Principles and Practice of Infectious Diseases. 2nd edn. Boston, Little Brown, 1985; pp. 1620-1625.

2. Tobin MJ, Grenvik A. Nosocomial lung infection and its diagnosis. Crit Care Med 1984; 12: 191-199.

3. Chastre J, Viau F, Brun P, et al. Prospective evaluation of the protected specimen brush for the diagnosis of pulmonary infections in ventilated patients. Am Rev Respir Dis 1984; 130: 924-929.

4. Baughman RP, Thorpe JE, Staneck J, Rashkin M, Frame PT. Use of the protected specimen brush in patients with endotracheal or tracheostomy tubes. Chest 1987; 91: 233-236.

5. Fagon JY, Chastre J, Hance AJ, et al. Detection of nosocomial lung infection in ventilated patients: use of a protected specimen brush and quantitative culture techniques in 147 patients. Am Rev Respir Dis 1988; 138: 110116.

6. Torres A, Puig de la Bellacasa J, Xaubet A, et al. Diagnostic value of quantitative cultures of bronchoalveolar lavage and telescoping plugged catheters in mechanically ventilated patients with bacterial pneumonia. Am Rev Respir Dis 1989; 140: 306-310.

7. Pham LH, Brun-Buisson C, Legrand P, et al. Diagnosis of nosocomial pneumonia in mechanically-ventilated patients: comparison of a plugged telescoping catheter with the protected specimen brush. Am Rev Respir Dis 1991; 143: 1055-1061.

8. Chastre J, Fagon JY, Soler P, et al. Diagnosis of nosocomial bacterial pneumonia in intubated patients undergoing ventilation: comparison of the usefulness of bronchoalveolar lavage and the protected specimen brush. Am J Med 1988; 85: 499-506.

9. Solé J, Rodríguez de Castro F, Rey A, Martín JC, Cabrera P. Usefulness of microscopic examination of intracellular organisms in lavage fluid in ventilator associated pneumonia. Chest 1994; 106: 889-894.

10. Rodríguez de Castro F, Solé J, Lafarga B, Caminero J, González B, Manzano JL. Reliability of the bronchoscospic protected catheter brush in the diagnosis of pneumonia in mechanically-ventilated patients. Crit Care Med 1991; 19: 171-175.

11. Solé J, Rodríguez de Castro F, Caminero J, Bordes A, Manzano JL. Comparative efficacy of bronchoalveolar lavage and telescoping plugged catheter in the diagnosis of pneumonia in mechanically-ventilated patients. Chest 1993; 103: 386-390.

12. Niederman MS, Torres A, Summer W. Invasive diagnostic testing is not needed routinely to manage suspected ventilator-associated pneumonia. Am J Respir Crit Care Med 1994; 150: 565-569.

13. Chastre J, Fagon JY. Invasive diagnostic testing should be routinely used to manage ventilated patients with suspected pneumonia. Am J Respir Crit Care Med 1994; 150: 570-574.

14. Wimberley N, Faling LJ, Bartlett JG. A fiberoptic bronchoscopy technique to obtain uncontaminated lower airway secretions for bacterial culture. Am Rev Respir Dis 1979; 119: 337-343.

15. Rodríguez de Castro F, Solé J, Elcuaz R. Quantitative cultures of protected brush specimens and bronchoalveolar lavage in ventilated patients without suspected pneumonia. Am J Respir Crit Care Med 1994; 149: 320-323.

16. Griner F, Mayewski RJ, Mushlin AI, Greenland P. Selection and interpretation of diagnostic tests and procedures: principles and applications. Ann Intern Med 1981; 94: 553-560.

17. Fagon JY, Chastre J, Trouillet JL, et al. Evaluation of patients suspected of having nosocomial pneumonia: are invasive procedures necessary for accurate decision making? Am Rev Respir Dis 1988; 137: A219 (Abstract).

18. Fagon JY, Chastre J, Hance AJ, Domart Y, Trouillet JL, Gibert C. Evaluation of clinical judgement in the identification and treatment of nosocomial pneumonia in ventilated patients. Chest 1993; 103: 547-553.

19. Unertl KE, Lenhart FP, Forst H, Peter K. Systemic antibiotic treatment of nosocomial pneumonia. Intensive Care Med 1992; 18(S): 28-34.

20. Pennington JE. Approach to therapy of respiratory infections in the critical care setting. Semin Respir Infect 1990; 5: 226-232.

21. Rello J, Ausina V, Castella J, Net A, Prats G. Nosocomial respiratory tract infections in multiple trauma patients: influence of level of consciousness with implications for therapy. Chest 1992; 102: 525-529.

22. Moine P, Vercken JB, Chevret S, Chastang C, Gajdos $\mathrm{P}$, and the French Study Group for Community-Acquired Pneumonia in the Intensive Care Unit. Severe community-acquired pneumonia: etiology, epidemiology and prognosis factors. Chest 1994; 105: 1487-1495.

23. Kuning CM. Problems in antibiotic usage. In: Mandell GL, Douglas RG, Bennett JE, eds. Principles and practice of Infectious Diseases. 2nd edn. New York, Churchill Livingstone, 1985; 301-307.

24. Fagon J-Y, Chastre J, Domart Y, et al. Nosocomial pneumonia in patients receiving continuous mechanical ventilation: prospective analysis of 52 episodes with use of a protected specimen brush and quantitative culture techniques. Am Rev Respir Dis 1989; 139: 877-884. 